\title{
New hardware for a streamlined cryo focused ion beam milling workflow
}

Sebastian Tacke ${ }^{1}$, Zhexin Wang ${ }^{2}$, Michael Grange ${ }^{2}$ and Stefan Raunser ${ }^{3}$

${ }^{1}$ Department of Structural Biochemistry, Max Planck Institute of Molecular Physiology, Otto-Hahn-Str. 11, 44227 Dortmund, Germany, United States, ${ }^{2}$ Max Planck Institute of Molecular Physiology, Dortmund, Germany, ${ }^{3}$ Max Planck Institute of Molecular Physiology, United States

Electron tomography at cryogenic temperatures (cryo-ET) offers the unique possibility to structurally analyse biological macromolecules in their native cellular environment [Grange2017, Bartesaghi2012]. Using this method, it is even possible to determine protein structures at near-atomic resolution by applying sub-volume averaging techniques [SChur2016].

One prerequisite for electron cryo microscopy is that the sample is thinner than the inelastic meanfree-path length of electrons (approximately $350 \mathrm{~nm}$ for $300 \mathrm{keV}$ electrons [Vulovic2013]. Thicker samples result in an increased number of inelastically scattered electrons, which decreases the quality of the image. The majority of biological samples (e.g. eukaryotic cells or tissues) are too thick for cryo-ET and need to be thinned.

Cryo-ultramicrotomy is one approach that can be used to reduce specimen thickness. Here, the sample is cut into ribbons of vitreous sections that are thin enough to be imaged by cryo-ET [AlAmoudi2004]. However, this method is technically demanding and prone to sample-altering artefacts such as knife marks, compression or crevasses [Al-Amoudi2005]. Another approach uses a focused ion beam under cryogenic conditions (cryo-FIB) in a scanning electron microscope (SEM) to thin samples [Marko2007, Rigort2010]. In recent years, numerous studies have demonstrated the suitability of this technique for preparing biological samples for cryo-ET without the artefacts known from cryoultramicrotomy [Hagen2015, Mahamid2016].

Current best-practice protocols for cryo-FIB milling include: i) vitrification of the sample, ii) identification of regions-of-interest, iii) rough ablation of the surrounding sample material and iv) polishing of the target region, leaving a thin lamella ready for cryo-ET [Meideiros2018]. However, the entire procedure involves multiple handling, transfer, milling and imaging steps. During these steps, samples need to be handled with forceps and it is difficult even for highly experienced practitioners not to destroy the thin and fragile lamellae. Moreover, since the vitrified sample needs to be cooled constantly to avoid hexagonal ice formation (devitrification), it is especially prone to any kind of ice contamination. 
Contamination of polished lamellae is particularly disruptive to cryo-ET as ice crystals often obscure the view on features otherwise visible in the lamellae. It is even possible that ice crystals (frost contamination) conceal the entire lamella, which prevents any meaningful data acquisition. Besides the transfer, which bears a high risk of exposing the sample to water, a potential source of (amorphous) ice contamination is the cryo-FIB/SEM itself. Despite the high vacuum inside the SEM, water molecules from the residual gas inside the microscope chamber can still deposit on the sample that is kept well below $-140{ }^{\circ} \mathrm{C}$ to avoid devitrification. Depending on the partial pressure of water inside the chamber, this effect can be severe and becomes especially problematic when samples reside in the SEM for several hours.

To obtain optimal lamellae for cryo-ET and to unfold the full strength of automated cryo-FIB milling, the following major hurdles need to be overcome: First, all types of frost contamination need to be reduced to a minimum. Second, all amorphous ice contamination inside the microscope needs to be avoided. Third, the handling of the lamellae must be streamlined to avoid lamella cracking.

Here, we present an integrated workflow that addresses and overcomes all three bottlenecks. In a first step, we implemented a specially designed glove box and a high vacuum cryo transfer system into the current workflow to reduce frost contamination during handling and transfer (Figure 1). Secondly, we tremendously reduced the contamination rates inside the cryo-FIB/SEM by the installation of a stage heater, a cryo-shield and a cryo-shutter (Figure 2). Thirdly, we implemented new tools to simplify the sample preparation and handling.

We show that the new hardware increases the overall throughput of high-quality lamella production, enabling new types of experiments, which are considered infeasible with current setups.

\section{Acknowledgements}

We thank Roger Wepf for many fruitful discussions and the exchange of ideas. We thank Philipp Erdman, Sven Klumpe and Jürgen Plitzko für the fruitfull cooperation. This work was supported by funding from the Max Planck Society (to S.R. and J.P.). M.G. is supported by an EMBO Long-Term Fellowship. 

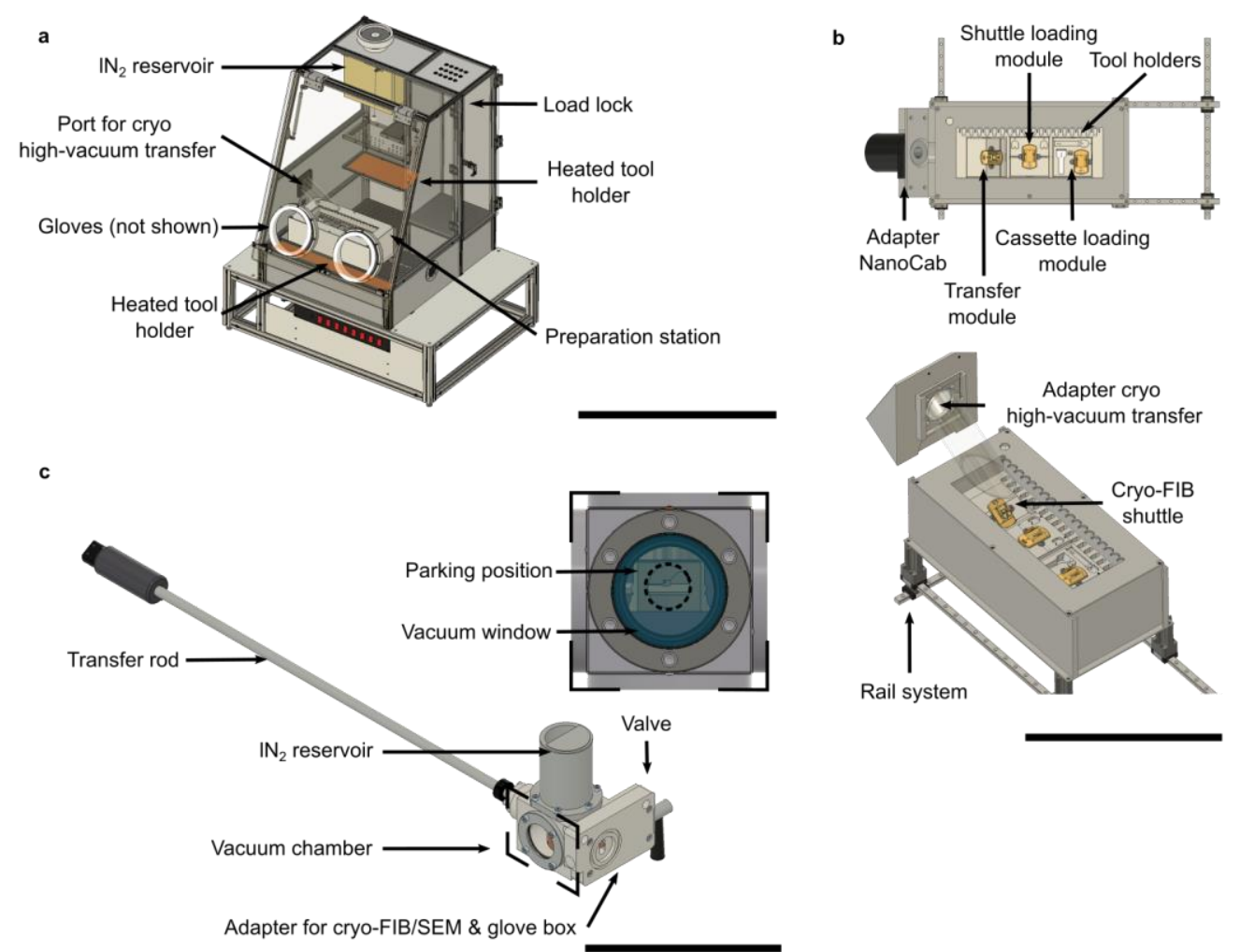

Figure 1. Schematic of the glove box, the preparation station, and the high vacuum cryo transfer system. a) The glove box enables convenient sample handling in an anhydrous environment. To reduce the humidity to a minimum, the glove box is purged during operation with nitrogen and a cold trap is installed in the back of the $1 \mathrm{~N} 2$ reservoir. $1 \mathrm{~N} 2$ can be taken from this reservoir for applications inside the glove box. For convenient handling, heated tool holders are installed. A load lock enables the insertion of tools without breaking the humidity barrier. Scale bar, $600 \mathrm{~mm}$. b) A new preparation station guarantees a comfortable and secure handling of the sample material. The preparation station consists of four parts: the liquid nitrogen bath, the preparation modules, the adapter for the transfer systems and the adapter for the NanoCab. All modules can be re-arranged inside the liquid nitrogen bath according to the preferences of the operator and are designed for left- and right-handed persons. Clipped autoloader C-clip rings can be loaded onto the cryo-FIB shuttle or to the autoloader cassette using the corresponding modules. Also clipping is possible in the preparation station but is not shown in this overview. The preparation station is mounted onto a rail system to ensure highest flexibility inside the glove box. Scale bar, $400 \mathrm{~mm}$. c) The high vacuum cryo transfer system consists of a transfer rod for picking up the cryo-FIB shuttle, a vacuum chamber, liquid nitrogen reservoir, a valve and the adapter for the cryo-FIB/SEM and glove box. The sample can be picked up by the transfer rod and is inserted in the vacuum chamber. Inside the vacuum chamber the cryo-FIB shuttle is placed in a parking position. Here, the shuttle is actively cooled by the 1N2 reservoir. The liquid nitrogen acts also as a cryo-pump and maintains the vacuum level, while the high vacuum cryo transfer is disconnected from the pumps. The $1 \mathrm{~N} 2$ volume holds for approximately 30 min. After the shuttle is positioned in the parking position, the valve towards the glove box is closed and the high vacuum cryo transfer is evacuated. Thereafter, the valve at the transfer system is closed and the sample can be transferred to the cryo-FIB/SEM. Scale bar, $300 \mathrm{~mm}$. 

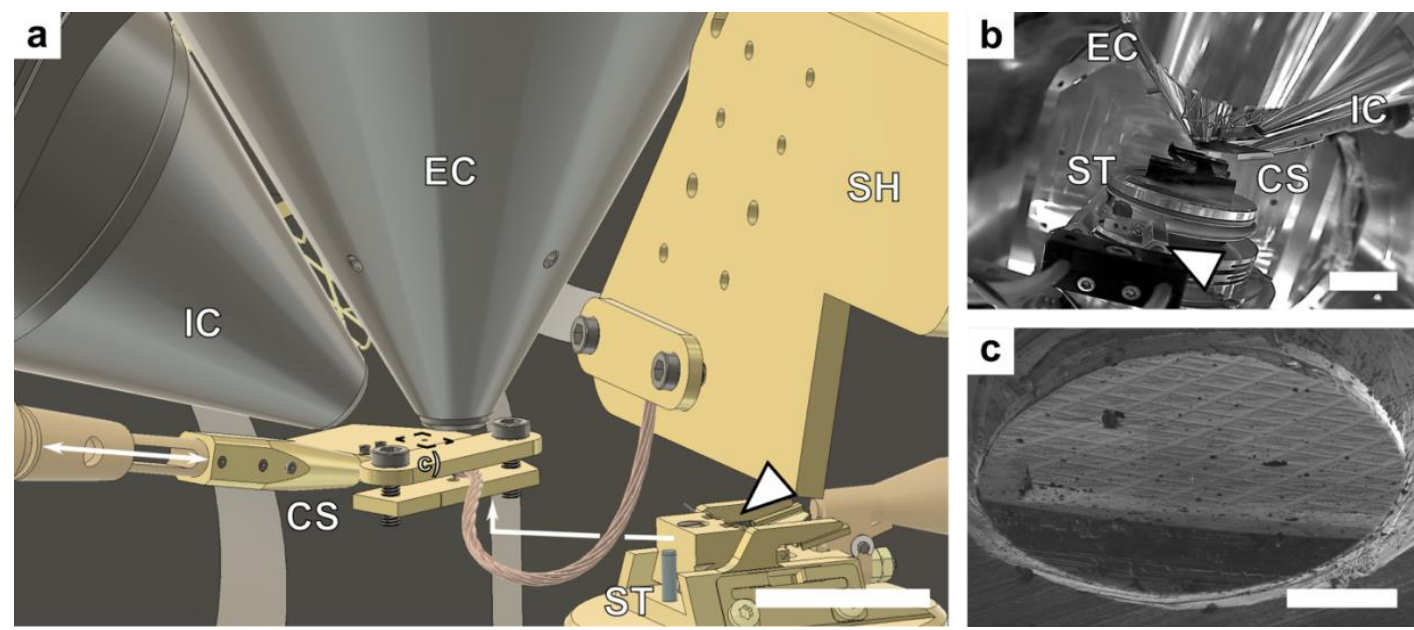

Figure 2. Cryo-shield and cryo-shutter. a) Schematic of the cryo-FIB/SEM chamber. The installed cryoshield (SH) is installed next to the electron column (EC) and the ion column (IC). The total surface area of the cryo-shield ( $\mathrm{SH}$ ) is approximately $880 \mathrm{~cm} 2$. Assuming that the strength of the pump is $\sim 151 \mathrm{~cm}-2$ $\mathrm{s}-1$, the estimated total pumping speed is $\sim 13,0001 \mathrm{~s}-1$. To reduce the partial pressure in the vicinity of the sample even further, a cryo-shutter (CS) can be inserted during milling (white arrow). The shuttle with the autoloader grids (white triangle) is moved via the cryo stage (ST) right below the cryo-shutter. Two different cryo-shutter types were tested. Cryo-shutter Type II covers most of the grid reducing the contamination rate even further compared to the cryo-shutter Type I. Scale bar, $25 \mathrm{~mm}$. b) Camera image from the back of the chamber. The cryo-shutter covers the autoloader grids almost completely. The white triangle indicates the position of the stage heater (not installed in this image). Scale bar, $25 \mathrm{~mm}$. c) Ion image of the cryo-shutter Type II, showing the EM grid through the shutter hole. The diameter of the shutter hole is approximately $1 \mathrm{~mm}$ to ensure a complete protection of the grid. For electron imaging, the shutter is removed automatically. With these improvements, the sample can be prepared and stored in the milling position until imaging without amorphous ice contamination. Scale bar, $300 \mu \mathrm{m}$.

\section{References}

[Grange 2017] Grange, M, Vasishtan, D., \& Grünewald, K. Cellular electron cryo tomography and in situ sub-volume averaging reveal the context of microtubule-based processes. J. Struct. Biol. 197, 181-190 (2017).

[Bartesaghi 2012] Bartesaghi A. et al. Secondary Structure Determination by Constrained Single-Particle Cryo-Electron Tomography. Structure 20, 2003-2013 (2012).

[Schur 2016] Schur, F. K. M. et al. An atomic model of HIV-1 capsid-SP1 reveals structures regulating assembly and maturation. Science 353, 506-508 (2016).

[Vulovic 2013] Vulovic, M. et al. Image formation modelling in cryo-electron microscopy. J. Struct. Biol. 183, 19-32 (2013).

[Al-Amoudi 2004] Al-Alamoudi, A., Norlen, L.P.O., \& Dubochet J. Cryo-electron microscopy of vitreous sections of native biological cells and tissues. J. Struct. Biol. 148, 131-135 (2004) 
[Al-Amoudi 2005] Al-Alamoudi, A., Studer, D. \& Dubochet, J. Cutting artefacts and cutting process in vitreous sections for cryo-electron microscopy. J. Struct. Biol. 150, 109-121 (2005).

[Marko 2007] Marko, M. et al. Focused-ion-beam thinning of frozen-hydrated biological specimens for cryo-electron microscopy. Nat. Methods 4, 215-217 (2007).

[Rigort 2010] Rigort, A. et al. Micromachining tools and correlative approaches for cellular cryo-electron tomography. J. Struct. Biol. 172, 169-179 (2010).

[Mahamid 2016] Mahamid J. et al Visualizing the molecular sociology at the HeLa cell nuclear periphery. Science 351, 969-972 (2016).

[Medeiros 2018] Medeiros, J.M. et al. Robust workflow and instrumentation for cryo-focused ion beam milling of samples for electron cryotomography. Ultramicroscopy 190, 1-11 (2018). 